ARGUMENTS

\title{
TRANSFORMATIONS MÉDIATIQUES : QUELQUES RÉFLEXIONS SUR LA NOTION DE «SÉRIE CULTURELLE » CHEZ ANDRÉ GAUDREAULT ET PHILIPPE MARION
}

Jan Baetens, Johan Callens, Michel Delville, Bertrand Gervais, Heidi Peeters, Robyn Warhol \& Myriam Watthee-Delmotte

\section{À la recherche de concepts transdisciplinaires}

S'il n'existe pas, aujourd'hui, de modèle ou de paradigme unique ou dominant en études littéraires, force est de constater que la dimension historique est, de nouveau, partout très présente (pour un aperçu, voir Vaillant 2010). Quelle que soit l'orientation méthodologique ou théorique des chercheurs en question, l'intérêt pour l'histoire est manifeste. Cependant, l'histoire littéraire que l'on pratique de nos jours n'est plus seulement celle des textes et des auteurs, ni celle des institutions qui les rendent possibles (l'édition, l'enseignement, la politique culturelle, entre autres). Elle inclut toujours davantage une dimension médiologique, par quoi il convient d'entendre à la fois ce qui relève du médium (soit la base matérielle du support qui permet la production, la distribution, la transformation des signes) que ce qui renvoie au média (soit les pratiques, les conventions et les idées qui se construisent autour de certains emplois signifiants et institutionnellement 
cadrés du médium). Il existe une grande variété de termes et de concepts qui tous explorent la même tension entre médium et média ${ }^{1}$, mais c'est bien le croisement de la littérature (en tant que corpus et pratique institutionnalisée), du média et de l'histoire que l'on retrouve au cœur des recherches les plus stimulantes d'aujourd'hui'. Dans une certaine mesure on pourrait d'ailleurs en dire autant des analyses de la culture en général, où s'est récemment imposée la position de la media archeology ou archéologie médiatique (Baetens, 2014).

Le caractère de plus en plus interdisciplinaire de tous ces travaux fait que la publication d'un ouvrage portant sur l'histoire du cinéma peut se révéler capitale pour des chercheurs en littérature, sans que personne ne doive renoncer à la spécificité de son propre champ. Interdisciplinarité n'égale pas nécessairement convergence ou hybridation, mais occasion de creuser l'articulation d'approches diverses, à la fois autonomes et interdépendantes. Dans cette perspective, La fin du cinéma ? (Gaudreault \& Marion, 2013) devrait retenir l'attention de tout historien de la littérature. Les pages qui suivent tenteront de rassembler quelques aspects clé de La fin du cinéma?, afin d'en dégager quelques enjeux pour une histoire de la littérature qui intègre la notion de média (et pas seulement de médium, comme on l'a toujours fait dans les études sur l'histoire du livre et de l'édition).

\section{Les concepts clé de l'analyse du cinéma selon Gaudreault et Marion}

Précisons-le d'emblée : Gaudreault et Marion se limitent expressément à la dimension médiatique du cinéma en général, à l'exclusion de ce que l'on trouve d'habitude dans les histoires du cinéma : les titres des films et les noms des metteurs en scène à retenir. Avant de se pencher sur la manière dont ces deux auteurs envisagent l'histoire du cinéma en termes médiatiques, il importe donc de décrire de manière aussi claire et nette que possible la signification du concept de média qu'ils développent dans leur étude.

1 Dans le contexte américain, cette distinction n'est pas sans rappeler la différence entre media studies et medium theory, les premières axées sur les questions de médium (elles dominent logiquement dans les départements de communication), les secondes ouvertes sur les questions de média (elles sont représentées surtout dans les travaux de médiologie et des cultural studies. Pour un aperçu global, Meyrowitz (1985) \& MDRN (2013).

2 Pour plus de détails, voir respectivement du point de vue des études de la communication et des études littéraires, Baetens et al. (2014). 
Les réponses traditionnelles à ce type de question tendent à mettre en lumière deux éléments. D'une part la pellicule - d'où le danger, réel ou supposé, de l'apparition du numérique, qui se passe de la pellicule. D'autre part la salle de cinéma, où a lieu une projection, dans le noir, devant un public plus ou moins nombreux - d'où le danger comparable, en termes définitionnels, des modes de distribution et de consommation de l'objet cinématographique qui n'ont plus lieu dans ce type de salle : est-ce encore un film, ce qu'on regarde sur un écran soit domestique, soit transportable? Pareille définition, dont l'histoire du cinéma a multiplié les exemples ${ }^{1}$, n'est aujourd'hui plus tenable. Non seulement à cause de son essentialisme, mais aussi et surtout en raison des multiples démentis que l'évolution des matérialités cinématographiques lui a infligés : le cinéma n'a pas toujours été fait sur pellicule - il a pu être tourné en vidéo. De la même façon, il a souvent été montré en des lieux tout autres qu'une salle de cinéma - et ce dès le début même des premières séances cinématographiques, avec le clivage des systèmes antagonistes d'Edison (visionnement individuel) et des Lumière (projection en salle). Une autre définition, à la fois plus ouverte et plus dynamique, à la fois susceptible d'intégrer des éléments plus larges que la projection du film sur pellicule en salle obscure et capable d'évoluer elle-même dans le temps, est donc indispensable.

Mais comment La fin du cinéma? pense-t-il la notion de média, c'est-à-dire du média en général, la question particulière du cinéma n'étant qu'une application ou exemplification de cette nouvelle définition. Gaudreault et Marion ne se contentent pas de dire qu'un média est fonction de la manière dont s'institutionnalise la mise en œuvre d'un médium (soit : j'invente une machine, par exemple le cinématographe : médium, qui m'aide à faire des films, puis à inventer de nouvelles formes de production, de distribution et de réception : média), ils soulignent surtout que le binôme médium/média ne peut luimême se comprendre qu'à partir d'un cadre plus vaste, où l'on trouve aussi bien un amont, celui qu'ils nomment les séries culturelles, qu'un aval, que l'on pourrait désigner du concept de pratiques culturelles.

1 Elle est au cœur de la théorie dite du « dispositif », illustrée entre autres par un article devenu très influent de Baudry (1975). 
La fin du cinéma? insiste beaucoup sur la première notion, celle de série culturelle. Ce concept, très controversé mais dont l'emploi est en train de se répandre très vite ${ }^{1}$, pour Gaudreault et Marion :

La série culturelle est une création de l'historien : celuici saisit un thème, un savoir-faire culturel (un type de spectacle, un type de représentation plus ou moins lié à un dispositif, à un appareillage), dont il essaie de retracer et de comprendre le parcours identitaire à travers ses différentes mutations, par exemple : la féerie, le cirque, l'image projetée, l'image graphique, l'image animée, l'opéra (Gaudreault \& Marion, 2013, p. 251).

Une série culturelle, si on essaie de lire cette définition de manière aussi large et ouverte que possible, serait donc un certain type de faire (par exemple raconter une histoire ou essayer de reproduire la réalité) doté d'une « identité » culturelle et médiatique historiquement identifiable et qui peut prendre les formes matérielles et médiatiques les plus diverses (on peut raconter des histoires à l'aide de mots mais aussi à l'aide de sons ou d'images, tout comme on peut essayer de reproduire le réel, ou un aspect ou une partie du réel, au moyen d'instruments visuels ou verbaux, analogiques ou digitaux, concrets ou abstraits, et ainsi de suite). Une telle série, est-elle nécessairement, comme le pose La fin du cinéma?, une " création de l'historien »? L'identification et la caractérisation, y compris sur le plan terminologique, ne peuvent-elles émerger de la pratique ou des praticiens eux-mêmes, avant même que l'historien ne se penche sur la question ? Et l'existence d'une certaine conscience sérielle, pour diffuse qu'elle soit souvent, ne peut-elle pas contribuer au succès et à la diffusion de la série même ? Quant à l'institutionnalisation, n'est-elle pas aussi un facteur de figement autant que de relance? Sur ces questions, les avis divergent sans doute.

Quoi qu'il en soit, il est clair que, dans l'approche de Gaudreault et Marion, une série culturelle comprend (toujours ?) plusieurs médias et qu'inversement un média peut appartenir à plusieurs séries. La série « documentaire », par exemple, peut prendre la forme d'un reportage journalistique, illustré ou non, mais aussi celle d'un film muet. Quant au

1 Pour plus de détails sur le débat suscité par la « série culturelle », voir Albera et al. (2009). Pour une illustration du succès croissant de la notion, on peut se reporter au programme du séminaire de recherche InTru (Université François Rabelais de Tours) du cycle 2012-2013 : http://intru.hypotheses.org/283 
texte ou au film documentaire, il peut relever non seulement de la série « documentaire », mais aussi, et toujours par exemple, de la série « art ».

Si la série culturelle constitue en quelque sorte l'amont de toute analyse médiatique, la pratique culturelle, concept qui ne se trouve pas au centre des analyses panoramiques de La fin du cinéma?, en serait un possible aval. De telles pratiques (ici aussi, le pluriel est de rigueur) désignent la manière précise, variable dans le temps comme dans l'espace, dont un média s'intègre aux rapports et interactions des médias existants, puis la manière dont le média se « vit » dans un contexte social donné.

La définition de ce qu'est une série culturelle et de ses rapports avec les pratiques culturelles qui s'y trouvent liées, a déjà fait l'objet de nombreuses polémiques. Mais au-delà des questions de définition il importe de souligner ce qui est sans doute l'apport décisif de Gaudreault et Marion aux recherches en histoire médiatique. Le mérite essentiel du concept de série culturelle est d'avoir montré que les divers éléments du complexe série/média/médium/pratique sont en fait solidaires les uns des autres et que ce qui se dit et se pense du côté de la série culturelle, c'est-à-dire de ce qui se situe en amont aussi bien du média que du médium, a un réel impact sur la manière dont un média se pratique, et inversement, tant du point de vue synchronique que du point de vue diachronique.

Une série culturelle mélange et combine divers médias, certes, mais les rapports entre ces médias ne sont jamais neutres. D'un média à l'autre, la force et le poids respectifs ne sont pas les mêmes. La série culturelle « faire un récit » peut prendre la forme d'un roman ou d'un film, pour prendre l'exemple contemporain le plus banal qui soit, mais, du moins aux yeux des théoriciens de la « re-médiation », le récit cinématographique sera jugé préférable au récit romanesque, le premier ayant « amélioré » le second (Bolter \& Grusin, 1999). Corollairement, les diverses séries qui se croisent dans un média ne sont pas non plus à pied d'égalité. Dans le média « cinéma », par exemple, la série « raconter une histoire »a longtemps exercé un règne sans partage, toutes les autres séries ayant été refoulées sans trop de discussion aux marges du système (alors qu'il a toujours existé des usages moins narratifs, voire non-narratifs ou anti-narratifs du média). De la même façon que médium, média et pratiques culturelles changent dans le temps, la « construction » d'une série culturelle - et partant des liens entre médias et séries culturelles - ne bénéficie jamais que d'une stabilité passagère. 
Reprenons à cet égard les deux exemples que nous venons de mentionner. D'une part, l'idée qu'un récit gagne à être cinématographique plutôt que romanesque est une idée relativement récente (serait-il permis d'en situer l'émergence officielle en 1948, au moment où Alexandre Astruc met en circulation la notion de caméra-stylo - sans doute une rupture aussi franche, et symboliquement forte, ne rend guère compte de la complexité des changements historiques ? (Astruc, 1945)) et rien n'interdit de penser que le roman retrouvera un jour le prestige qui fut le sien (l'hypothèse a beau briller par son caractère peu plausible aujourd'hui, mais les exemples historiques d'une actualisation des formes « résiduelles ${ }^{1} »$ ne manquent pas). D'autre part, l'idée qu'un film est d'abord fait pour raconter une histoire est une conviction également fragile, d'abord parce que le cinéma des premiers temps n'était pas vraiment narratif (sa narrativité était « intrinsèque », liée au déroulement mécanique des images dans le temps. La narrativité « extrinsèque », résultat d'une intervention voulue sur les enregistrements, est ultérieure et n'a elle non plus rien d'éternel), ensuite parce que le cinéma de nos jours, ce cinéma « élargi » qui a migré vers d'autres supports matériels que celui de la projection en salle, se caractérise souvent par un certain fléchissement, si l'on peut dire, au moment du retour du cinéma d'attractions, de son programme narratif (pour un aperçu très fouillé, voir Shaw \& Weibel, 2003).

Or, si tout le monde accepte sans peine que les rapports entre séries culturelles et médias sont susceptibles de changer, il reste à expliquer pourquoi ces modifications se produisent et à quelle logique ils obéissent. Tout en attachant une réelle importance à l'analyse du médium comme substrat matériel et technique du média, Gaudreault et Marion rejettent catégoriquement le déterminisme technologique, qui n'est pas rare dans les recherches historiques sur les médias². Puisque le médium n'est qu'une partie, et une partie seulement, d'un jeu plus complexe qui engage aussi des éléments imaginaires, émotifs, historiques ou encore idéologiques, La fin du cinéma? refuse de confondre média et médium (ce qui nous paraît une définition assez logique du déterminisme technologique en contexte médiatique), puis de réduire un média à une seule série culturelle et vice versa (ce qui est une autre

1 Voir Acland (2007). La notion de « résiduel », qui fait partie de la triade dominant/ émergent/résiduel, est empruntée aux travaux de Raymond Williams (1977).

2 Toutes proportions gardées, la théorie de la re-médiation de Bolter et Grusin en serait elle aussi une illustration, malgré la distance que les auteurs prennent par rapport à McLuhan (accusé, à tort ou à raison, de déterminisme technologique). 
forme de déterminisme, bien entendu). Aux liens rigides entre média, médium et série, Gaudreault et Marion substituent une perspective à la fois généalogique (soucieuse de reconstruire l'évolution historique d'une forme médiologique) et archéologique (attentive aux ruptures qui caractérisent les transformations internes d'une telle forme). Dit autrement : ils cherchent à mettre au jour les principes généraux qui régissent et le passage d'une étape à l'autre (c'est le côté diachronique, ou si l'on préfère généalogique) et la loi qui explique à tel moment et à tel endroit, laisse comprendre pourquoi le média se pense, se construit, se vit comme il le fait (c'est le côté archéologique de l'analyse, qui conçoit les mutations historiques en termes de rupture : d'une étape à l'autre, il n'y a pas évolution ou transformation mais cassure et substitution d'un modèle sous-jacent à l'autre).

Pour ce qui est de la perspective généalogique de l'analyse, les auteurs reviennent en détail sur une proposition théorique qui les occupe depuis de longues années déjà. Formulée pour la première fois en 1999 (Gaudreault \& Marion, 2000), la théorie de la « double naissance » repose sur la distinction entre médium et média. Dans un premier temps (" première naissance »), une nouvelle technique ou un nouveau dispositif émerge, par exemple le cinématographe, machine à capter et à projeter des vues mobiles, mais son emploi ne donne pas encore lieu à des usages médiatiques au sens institutionnel du terme : on voit bien que quelque chose s'est passée en termes de médium, sans qu'il soit déjà possible de voir quel nouveau média pourrait s'en engendrer. Dans un second temps (« seconde naissance »), les usages du médium interagissent avec les médias existants pour faire surgir un nouveau média, ce qui fait comprendre rétrospectivement l'événement qu'a constitué l'apparition du nouveau médium : le cinématographe devient cinéma au bout d'une série de déplacements historiques, comme surtout la mise en place d'un réseau de salles exclusivement réservées à la projection de films, une mutation qui bouleverse la totalité des systèmes de production, de distribution et de réception du nouveau médium devenu média. Dans La fin du cinéma?, Gaudreault et Marion reviennent en détail sur cette théorie, dont ils proposent ici une version élargie et nuancée. Logiquement, ils évoluent vers une théorie de la «multiple naissance », qui est aussi leur façon de répondre au lieu commun, lancé en fait dès la première apparition du médium, de la mort du cinéma.

Pour ce qui est de la perspective archéologique de l'analyse, essentielle à l'intelligence des raisons même du changement, les 
auteurs jugent prioritaire le rapport entre série culturelle et média, plus exactement, l'accent mis sur telle ou telle série culturelle plutôt que sur telle autre dans la pratique concrète d'un média (aspect archéologique). C'est ce qu'ils appellent le caractère " sérialo-centré » de leur lecture médiatique et dont l'analyse des métamorphoses actuelles du cinéma, ce média en crise à l'ère numérique, offre une belle illustration. En effet, aux yeux de Gaudreault et Marion, le post-cinéma qui est en train de se répandre aujourd'hui, hors pellicule, hors salle, hors récit, est toujours du cinéma, mais un cinéma dont la série culturelle dominante n'est pas celle d'hier, à savoir raconter une histoire en projetant des images mobiles résultant d'une prise de vue réelle, mais une tout autre série, présente dès le début du cinéma comme médium mais soigneusement écartée ou refoulée au moment de sa seconde naissance en tant que média, qui voit l'arrivée du cinéma narratif réaliste projeté en salle), à savoir celle de la vue animée. Dans la généalogie et l'archéologie du média, l'animation n'est donc pas quelque chose qui relève d'un changement de médium (le passage du film sur pellicule à la production d'images numériques), mais un changement de média, qui prend sa source dans un nouveau champ de force entre éléments provenant de séries culturelles et éléments provenant de pratiques médiatiques.

On l'aura compris, un des grands atouts méthodologiques de cette approche « sérialo-centrique » serait de se permettre de penser en dehors de la boîte des schémas et genres institutionnellement constitués en faveur d'une approche susceptible de nous éclairer sur la généalogie de la production cinématographique au travers d'une mise en rapport avec des séries non-cinématographiques ou encore d' " hyperséries" fondatrices telles que l' « animation ». Les limites d'une telle approche, aussi féconde soit-elle, sont principalement liées à la nécessité de maîtriser à la fois l'histoire du cinéma et celle de la série culturelle : on peut, par exemple, lire Méliès au travers de la féerie mais encore fautil dénicher un historien du cinéma « qui ait acquis une connaissance suffisante de l'histoire de la féerie, bien en amont de l'apparition du cinématographe, pour renouveler réellement le point de vue » (Albera et al., 2009).

\section{Changements médiatiques, changements littéraires}

La fin du cinéma? n'est pas seulement un livre sur le cinéma, il est aussi un livre sur la notion de média. En ce sens, il représente une publication essentielle pour les chercheurs en histoire littéraire, où les 
débats méthodologiques demeurent très vifs, surtout en ce moment de tournant médiatique de la littérature.

Le premier effet du livre de Gaudreault et Marion est évidemment d'apporter de l'eau au moulin des « littéraires médiologues ». Avant d'être un art, une tradition, un objet de musée, voire un instrument politique et social, la littérature est un média, plus exactement une forme d'expression dont la dimension médiatique est fondamentale. Mais de quel média s'agit-il ? En effet, ce que nous appelons littérature est un terme qui coiffe des réalités hétérogènes - de l'épopée antique au roman graphique, du slam aux livres d'art, de la poésie visuelle au théâtre, des hypertextes arborescents aux manifestes agit-prop photocopiés -, à tel point que les bases médiatiques de la littérature posent au moins autant de problèmes qu'elles n'en résolvent.

Grâce à La fin du cinéma ?, il est possible d'apporter plusieurs éclaircissements. Comme de manière très générale la définition d'un média ne dépend pas mécaniquement du recours à tel ou tel médium, il ressort des analyses de Gaudreault et Marion qu'un média peut non seulement survivre à des changements parfois très radicaux à hauteur du médium engagé, mais qu'il peut s'accommoder non moins aisément de la fusion ou combinaison de plusieurs médiums sans pour autant que cette interaction ne fasse nécessairement surgir un fatras postgénérique indifférencié identifié par les auteurs comme le « Grand Intermédia » (65) du divertissement hyper-connecté. De la même manière, Gaudreault et Marion établissent qu'un média est la conséquence d'un choix historique, celui de la série culturelle qui l'articule et lui donne sens en profondeur, et que comme tel son statut est par définition instable. Un média peut migrer d'une série culturelle à l'autre et partant renaitre sous une forme différente, même s'il n'est pas toujours possible d'identifier la nature exacte du changement : est-ce le média qui change ou estce que l'auteur change de média et, ce faisant, de statut auctorial ? Est-ce la variabilité même des processus médiatiques ? Des principes de marketing ? La facilité de l'emploi ? La législation ? L'appropriation d'un média par ses publics ? Le dynamisme intrinsèque des médias comme faits historiques condamne sans doute ce genre de questions à rester sans réponse définitive. Une étude plus poussée du changement médiatique devra toutefois se donner pour but de donner des réponses précises à ces questions.

La littérature n'arrête pas de s'enrichir de nouvelles formes médiatiques : aujourd'hui, l'élargissement au roman graphique en fournit un exemple spectaculaire. Mais il arrive aussi qu'elle en perde. 
Le théâtre par exemple, solidement ancré dans le champ littéraire depuis un grand nombre de siècles, a aujourd'hui pris son indépendance, pour se rapprocher, sans bien sûr s'y dissoudre tout à fait, d'un nouvel ensemble, celui de la "performance », où il tend à se confondre avec le champ de la danse, au moins en Europe (" arts de la scène » ou «spectacle vivant» sont des termes beaucoup plus étroits, qui ne rendent pas suffisamment compte d'une mutation beaucoup plus constitutive, dont témoigne la généralisation de labels comme "performing arts ", beaucoup plus larges que les seuls " arts de la scène »). Le théâtre contemporain est du reste un bel exemple de l'hybridation de plus en plus forte des médias, l'ancienne hiérarchie des reprises médiatiques, où le théâtre servait d'intermédiaire entre le roman (forme légitime) et l'adaptation cinématographique (forme populaire), étant remplacée par un système beaucoup plus fluide où les différences entre écriture dramatique et écriture cinématographique peuvent se brouiller.

La fin du cinéma? aide à voir plus clair dans ces chassés-croisés incessants. Ce qui rapproche ou disjoint des médias n'est pas tant ce qui les sépare ou les unit sur le plan du médium que ce qui les distingue ou les confond en termes de série culturelle, ou si l'on préfère de la fonction culturelle que l'on veut mettre en avant (Gaudreault et Marion soulignent à juste titre que cette préférence est une préférence historiquement et culturellement construite). Bref, si la littérature peut d'ores et déjà s'ouvrir à la bande dessinée, c'est parce qu'à l'intérieur du média littéraire, au sens traditionnel du mot, on privilégie aujourd'hui la série culturelle « faire un récit »- et peu importe alors que ce récit soit verbal ou visuel, distant ou immersif, cognitivement actif ou physiquement interactif. De même, pour que le théâtre puisse se détacher de la « littérature » (comprise dans une acception restrictive qui exclut la poésie, l'essai et tous les modes d'écriture qui s'écartent du récit comme le portrait ou le Nouveau roman par exemple), il est indispensable qu'il renonce en partie à sa base narrative, comme l'ont bien étudié les tenants du théâtre post-dramatique (Lehmann, 2002).

Quoi qu'il en soit, l'unité comme la diversité, voire l'hétérogénéité du média littéraire peuvent maintenant se penser de manière plus nette. $\mathrm{Du}$ point de vue synchronique, la critique du déterminisme technologique, qui aligne le média sur le médium, doit se prolonger d'une critique de la monoculture sérielle, qui enferme un média dans une seule série culturelle. Suite à Gaudreault et Marion, il convient de poser fermement qu'un média structuré par les attentes d'une série culturelle donnée peut fort bien être « happé » par une série différente. 
À l'intérieur d'un discours donné, au sens foucaldien du terme, les agents sont amenés à suivre les lois de ce discours, quand bien même des stratégies de résistance sont toujours à l'œuvre, selon la dynamique «paratopique » mise en évidence par Dominique Maingueneau (2004). Du point de vue diachronique, la théorie de la double naissance, puis de la re-naissance d'un média ne doit pas être conçue sur le modèle linéaire de la re-médiation, où le nouveau se substitue à l'ancien (non en le « tuant », certes, mais en l'enrichissant et en le complétant), mais comme une manière de sédimentation où chaque nouvelle couche provoque et structure de nouvelles interactions entre ce qui existe déjà.

La fin du cinéma? oblige aussi à repenser la fin de la littératureun des thèmes les plus lancinants dans le débat sociétal sur la littérature (Todorov, 2007 ; Compagnon, 2007 ; Jouve, 2010 ; Schaeffer, 2011). La différence radicale du cinéma et de la littérature saute ici aux yeux, ce qui est la preuve qu'en dépit de certaines convergences, les unes internes (avec l'accentuation de la fonction narrative), les autres externes (avec la quasi-fusion des champs littéraire et cinématographiques en tant que fournisseurs de « contenus » de la cour des grands groupes médiatiques, voir Collins (2010) et Thompson (2010)), littérature et cinéma sont des médias indépendants, mais leur interdépendance en termes de contenus est forte. Certes, tant le cinéma que la littérature traversent régulièrement des périodes de crise, où se meurt une certaine idée de ces médias, mais il naît toujours de nouvelles configurations. Toutefois, la pensée de la fin ou de la mort du média obéit de part et d'autre à des préoccupations on ne peut plus différentes. Au cinéma, ce qui paraît toujours menacé de disparition, c'est le média même, non sa diffusion ou son succès. Même ceux qui redoutent que le cinéma de demain n'ait plus rien à voir avec le cinéma d'aujourd'hui, c'est-à-dire d'hier car souvent nos idées sur le média sont en retard par rapport aux réalités médiatiques, savent que le monde ne manquera jamais ni d'images mobiles ni de possibilités de production et de réception de ces images. Par contre, ce qui inquiète en littérature n'est pas l'évaporation du média mais le recul social de l'institution (tout le monde écrit, mais personne ne lit plus, et ceux qui le font encore n'en tirent aucun profit en termes de distinction), puis l'incapacité du média à dépasser les contraintes de l'ancien médium (le livre résiste au zapping généralisé de la culture moderne tout comme il abuse de cette ressource devenue introuvable qu'est le loisir).

L'exemple de La fin du cinéma? montre cependant que l'analyse médiatique n'a rien à gagner de slogans apocalyptiques. La meilleure façon de s'interroger sur le thème éternel de la fin reste sans aucun doute 
l'application d'un peu plus de théorie médiatique. Pareille théorie, en effet, porte à conséquence : le regard sur le passé permet la redéfinition du présent et partant la nouvelle invention du futur.

\section{Références}

Acland, C.R. (Éd.). (2007). Residual Media. Minneapolis : University of Minnesota Press.

Albera, F., Boillat, A., Carou, A., \& Le Forestier, L. (2009). Pour une nouvelle histoire du cinématographe : Cinq questions à André Gaudreault. 1895. Mille huit cent quatre-vingt-quinze, 57, 9-16. Disponible à: http://1895.revues.org/4005

Astruc, A. (1948). Naissance d'une nouvelle avant-garde. L'Écran français.

Baetens, J. (2014). Le médium n'est pas soluble dans les médias de masse. Hermès, $70,40-45$.

Baetens, J., Callens, J., Delville, M., Gervais, B., Peeters, H., Warhol, R., \& WatthéeDelmotte, M. (2014). Literature and Media Innovation. A Brief Research Update on a Genre/Medium Project. Germanisch Romanische Monatsschrift, 64(4), 485-492.

Baudry, J.-L. (1975). Le dispositif : approches métapsychologiques de l'impression de réalité. Communications, 23, 56-72.

Bolter, J.D., \& Grusin, R. (1999). Remediation. Understanding New Media. Cambridge, MA : MIT Press.

Collins, J. (2010). Bring on the Books for Everybody. Chapel Hill, NC : Duke University Press.

Compagnon, A. (2007). La littérature, pour quoi faire? Paris : Collège de France/ Fayard.

Gaudreault, A., \& Marion, Ph. (2000). Un média naît toujours deux fois... Sociétés \& Représentations, 9, 21-36.

Gaudreault, A., \& Marion, Ph. (2013). La fin du cinéma? Paris : Colin.

Jouve, V. (2010). Pourquoi étudier la littérature? Paris : Colin.

Lehmann, H.T. (2002). Le théâtre postdramatique. Paris : L'Arche.

Maingueneau, D. (2004). Le discours littéraire. Paratopie et scène d'énonciation. Paris : Armand Colin.

MDRN. (2013). Modern Time. Literary Change. Leuven : Peeters.

Meyrowitz, J. (1985). No Sense of Place. New York : Oxford University Press.

Schaeffer, J.-M. (2011). Petite écologie des études littéraires - Pourquoi et comment étudier la littérature? Paris : Thierry Marchaisse.

Shaw, J., \& Weibel, P. (Éd.). (2003). Future Cinema: The Cinematic Imaginary after Film. Cambridge, MA : MIT Press.

Thompson, J.B. (2010). Merchants of Culture. London : Polity Press.

Todorov, T. (2007). La littérature en péril. Paris : Flammarion.

Vaillant, A. (2010). L'Histoire littéraire. Paris : Colin.

Williams, R. (1977). Marxism and Literature. New York : Oxford University Press. 